\title{
Evaluation of the Exponential Integral for Large Complex Arguments
}

\section{John Todd}

Two methods of evaluating the exponential integral for (large) complex arguments are discussed. It is shown that the Laguerre quadrature method is more efficient than the asymptotic expansion. Examples are given to show the practicability of the Laguerre method.

\section{Introduction}

An extensive table of the exponential integral

$$
E_{1}(z)=\int_{z}^{\infty}\left(e^{-u} / u\right) d u \quad z=x+i y
$$

has been prepared by the National Bureau of Standards $[1] ;{ }^{1}$ the introduction to the table gives a precise definition of this function. This table covers the range $|x| \leq 20,|y| \leq 20$, with arguments variously spaced. In order to compute $E_{1}(z)$ outside this range, (or within this range, at points where interpolation is awkward, which are those with comparatively large argument), several methods can be considered. We examine two in detail and recommend the Laguerre quadrature, which is certainly practicable in the case of isolated arguments.

\section{Asymptotic Series}

The first of these is the use of the asymptotic series (see e. g., [1]),

$$
E_{1}(z) \sim e^{-z}\left(\frac{1}{z}-\frac{1 !}{z^{2}}+\frac{2 !}{z^{3}}-\frac{3 !}{z^{4}}+\cdots\right) .
$$

The remainder after $n$ terms in the expansion for $e^{z} E_{1}(z)$ can be obtained by integration by parts and is

$$
R_{n}=(-)^{n} n ! e^{z} \int_{z}^{\infty} \frac{e^{-u}}{u^{n+1}} d u \text {. }
$$

If we put $u=z+\rho$, where $\rho$ is real, then

which can be estimated as

$$
R_{n}=(-)^{n} n ! \int_{0}^{\infty} \frac{e^{-\rho} d \rho}{[(x+\rho)+i y]^{n+1}}
$$

$$
\begin{array}{ll}
\left|R_{n}\right| \leq S_{n}=n !\left[x^{2}+y^{2}\right]^{-\frac{1}{2}(n+1)} & \text { if } x \geq 0 \\
\left|R_{n}\right| \leq T_{n}=n !|y|^{-n-1} & \text { if } x \leq 0,
\end{array}
$$

from which the asymptotic character of the series is evident. In fact,

$$
\frac{S_{n+1}}{S_{n}}=\frac{n+1}{\left(x^{2}+y^{2}\right)^{\frac{1}{2}}}
$$

and so $S_{n}$ decreases as $n$ increases until $n$ exceeds $\left(x^{2}+y^{2}\right)^{\frac{1}{2}}$. To obtain the least the least value of $S_{n}$ we have to take $n \div\left(x^{2}+y^{2}\right)^{\frac{1}{2}}=|z|$. For this value of $n$, we find, using Stirling's formula, that $S_{n}$ is about $(2 \pi / n)^{\frac{1}{2}} e^{-n}$. In the second case, the least value of $T_{n}$ is formally the same, but

${ }^{1}$ Figures in brackets indicate the literature references at the end of this paper. 
now $n \doteqdot|y|$. The decrease in the effectiveness of this representation as $z$ approaches the negative real axis is apparent from the estimates.

We note that the evaluation of the sums of the first $n$ terms of the series for $e^{z} E_{1}(z)$, as a polynomial in $z^{-1}$, by successive multiplication by $z^{-1}$ for instance, involves about $4 n$ real multiplications, $z$ being complex.

\section{Laguerre Quadrature}

The second method is the use of a Laguerre quadrature. We shall show that this is more efficient than the asymptotic series method just described.

We have

where

$$
e^{z} E_{1}(z)=I_{1}-i I_{2}
$$

$$
I_{1}=\int_{0}^{\infty} e^{-\rho \cdot} \frac{x+\rho}{(x+\rho)^{2}+y^{2}} \cdot d \rho, \quad I_{2}=\int_{0}^{\infty} e^{-\rho \cdot} \frac{y}{(x+\rho)^{2}+y^{2}} \cdot d \rho .
$$

It is well known [3] that, for any $n$,

$$
I=\int_{0}^{\infty} e^{-t} \cdot f(t) \cdot d t \doteqdot \Sigma \lambda_{i}^{(n)} f\left(x_{i}^{(n)}\right)=Q
$$

where the $x_{i}^{(n)}$ are the zeros of the Laguerre polynomial $L_{n}(t)$, and the $\lambda_{i}^{(n)}$ are the corresponding Christoffel numbers. The $x_{i}^{(n)}, \lambda_{i}^{(n)}$ have been tabulated for $n=1(1) 15, i=1(1) n$ by Salzer and Zucker [2].

Moreover, it is known [3] that for some $\xi, 0 \leq \xi \leq \infty$,

$$
R=[I-Q]=(n !)^{2} \frac{f^{(2 n)}(\xi)}{(2 n) !}
$$

We shall now estimate this error. It is convenient to handle the two integrals separately. We calculate the $2 n$th derivatives of

and find

$$
f_{1}(t)=\frac{x+t}{(x+t)^{2}+y^{2}}, \quad f_{2}(t)=\frac{y}{(x+t)^{2}+y^{2}}
$$

$$
\begin{aligned}
& R_{1}=(n !)^{2} r_{1}^{-2 n-1} \cos (2 n+1) \theta_{1}, \\
& R_{2}=(n !)^{2} r_{2}^{-2 n-1} \sin (2 n+1) \theta_{2},
\end{aligned}
$$

where the $\xi_{i}=\xi_{i}(x, y)$ satisfy $0 \leq \xi_{i} \leq \infty, r_{i}=\left[\left(x+\xi_{i}\right)^{2}+y^{2}\right]^{\frac{1}{2}}$, and $\theta_{i}$ is defined by $\cos \theta_{i}=\left(x+\xi_{i}\right) / r_{i}$, $\sin \theta_{i}=y / r_{i}$.

Hence

$$
\begin{array}{ll}
\left|R_{i}\right| \leq \frac{(n !)^{2}}{\left[x^{2}+y^{2}\right]^{n+\frac{1}{2}}} & x \geq 0, \\
\left|R_{\imath}\right| \leq \frac{(n !)^{2}}{|y|^{2 n+1}} & x \leq 0 .
\end{array}
$$

The difficulties that were noticed in section 2 , as $z$ approaches the negative real axis, are still present. As before, there is an optimum value of $n$, which is about $|z|$ in the first case, $|y|$ in the second. With this choice of $n$, the value of our bounds for the $R_{i}$ are about $2 \pi e^{-2 n}$ in each case.

The important fact to notice is that our estimate for the error committed by using an $n$-point Laguerre quadrature is approximately the square of that for the estimate of the error committed by neglecting all terms in the asymptotic series after the $n$ th. This, together with the fact that about the same number $(4 n)$ of real multiplications are required in both methods, indicates that the Laguerre method is more efficient. 


\section{Examples}

In order to demonstrate the practicability of the Laguerre method, and to show that our conclusions are not dependent on the crudity of our appraisals of the remainders, we give three examples in detail. We choose $n=5$, which is optimum in one but not in the others. For completeness, we repeat (from [2]) the values of $x_{i}{ }^{(5)}, \lambda_{i}{ }^{(5)}$.

\begin{tabular}{rlllll}
\multicolumn{3}{c}{$x_{i}^{(5)}$} & \multicolumn{3}{c}{$\lambda_{i}^{(5)}$} \\
0.26356 & 03197 & 18 & 0.52175 & 56105 & 83 \\
1.41340 & 30591 & 07 & .39866 & 68110 & 83 \\
3.59642 & 57710 & 41 & .07594 & 24496 & 817 \\
7.08581 & 00058 & 59 & .00361 & 17586 & 7992 \\
12.64080 & 08442 & 76 & .00002 & 33699 & 723858
\end{tabular}

The superscripts (5) will be dropped from now on.

We shall work to about 10 decimals throughout our examples. We begin with a case of $z$ real: $z=10$ and follow with $z=1+10 i$, and then $z=-10+5 i$; it is only in the last case that we are, with $n=5$, near the optimum number of terms.

(a) $z=10$.

In this case $f_{2}=0$, and we have only to deal with

$$
I_{1}=\int_{0}^{\infty} e^{-t} \cdot \frac{1}{(10+t)} \cdot d t \doteqdot \sum \lambda_{i} \cdot \frac{1}{10+x_{i}}=Q_{1} .
$$

A complete worksheet for this calculation follows. The sum $Q_{1}$ is cumulated on the machine, there being no reason to record individual products.

$$
\begin{aligned}
& \alpha_{i}=10+x_{i} \quad \alpha_{i}^{-1} \\
& \text { 10. } 2635603197 \quad 0.0974320771 \\
& 11.41340 \quad 30591 \quad .0876162872 \\
& 13.59642 \quad 57710 \quad .0735487412 \\
& \text { 17. } 08581 \quad 00059 \quad .0585281002 \\
& 22.6408008443 \quad .0441680490 \\
& Q_{1}=\sum \lambda_{i} \alpha_{i}^{-1}=0.0915633319
\end{aligned}
$$

The correct value of $e^{10} E_{1}(10)$ is 0.091563334 . The actual error is about $2 \times 10^{-9}$, whereas our estimate is $144 \times 10^{-9}$. Using five terms of the asymptotic series, we obtain the value 0.09164 . There is thus an actual error of about $8 \times 10^{-5}$, compared with an estimated error of $12 \times 10^{-5}$.

(b) $z=1+10 i$.

In this case we have

$$
e^{1+10 i} E_{1}(1+10 i)=I_{1}-i I_{2}
$$

where

$$
I_{1}=\int_{0}^{\infty} e^{-t} \cdot \frac{1+t}{(1+t)^{2}+100} \cdot d t \doteqdot \sum \lambda_{i} \cdot \frac{1+x_{i}}{\left(1+x_{i}\right)^{2}+100}=\sum\left(1+x_{i}\right)\left\{\frac{\lambda_{i}}{\left(1+x_{i}\right)^{2}+100}\right\}
$$

and

$$
I_{2}=\int_{0}^{\infty} e^{-t} \cdot \frac{10}{(1+t)^{2}+100} \cdot d t \doteqdot \sum \lambda_{i} \cdot \frac{10}{\left(1+x_{i}\right)^{2}+100}=\sum 10\left\{\frac{\lambda_{i}}{\left(1+x_{i}\right)^{2}+100}\right\}
$$


A complete work sheet for this calculation follows. It is convenient to compute the common factors $\gamma_{i}$, in braces \{\} above, and then obtain the sums $\sum \alpha_{i} \gamma_{i}$ and $\sum 10 \gamma_{i}$ by accumulation on the machine.

\begin{tabular}{|c|c|c|}
\hline$\alpha_{i}=1+x_{i}$ & $\beta_{i}=100+\alpha_{i}^{2}$ & $\gamma_{i}=\lambda_{i} / \beta_{i}$ \\
\hline 1. 263560320 & $101.59658 \quad 47$ & 0.0051355625 \\
\hline 2. $41340 \quad 3059$ & 105.8245143 & .0037672444 \\
\hline 4. 596425771 & 121. 1271299 & .0006269648 \\
\hline 8. 085810006 & $165.38032 \quad 35$ & .0000218391 \\
\hline 13. 640800844 & $286.07144 \quad 76$ & $.00000 \quad 00817$ \\
\hline
\end{tabular}

$$
\begin{aligned}
& \mathscr{R} e^{1+10 i} E_{1}(1+10 i) \doteqdot \sum \alpha_{i} \gamma_{i}=0.018640471 \\
& \mathscr{J} e^{1+10 i} E_{1}(1+10 i) \doteqdot-\sum 10 \gamma_{i}=-0.095516925
\end{aligned}
$$

The correct value of $e^{1+10 i} E_{1}(1+10 i)$ is $0.01864049-0.09551688 i$.

The actual error is of the order of $5 \times 10^{-8}$, whereas our estimate is about $144 \times 10^{-9}$. Using five terms of the asymptotic series gives

$$
0.018595-0.095600 i \text {. }
$$

There is thus an actual error of about $1 \times 10^{-4}$, compared with an estimated error of about $1.2 \times 10^{-4}$.

(c) $z=-10+5 i$

\begin{tabular}{rrrrrr}
\multicolumn{1}{c}{$\alpha_{i}=-10+x_{i}$} & \multicolumn{2}{c}{$\beta_{i}=25+\alpha_{i}^{2}$} & \multicolumn{2}{c}{$\gamma_{i}=\lambda_{i} / \beta_{i}$} \\
-9.736439680 & 119.7982576 & 0.00435 & 52855 \\
-8.586596941 & 98.7296470 & .00403 & 79645 \\
-6.403574229 & 66.0057629 & .00115 & 05428 \\
-2.914189994 & 33.4925033 & .00010 & 78378 \\
2.64080 & 0844 & 31.97382 & 91 & .00000 & 07309
\end{tabular}

$$
\begin{aligned}
& \mathscr{R} e^{-10+5 i} E_{1}(-10+5 i) \doteqdot \sum \alpha_{i} \gamma_{i}=-0.084757264 \\
& \mathscr{I} e^{-10+5 i} E_{1}(-10+5 i) \doteqdot-\sum 5 \gamma_{i}=-0.048261807
\end{aligned}
$$

The correct value of $e^{-10+5 i} E_{1}(-10+5 i)$ is

$$
-.08475749-0.04826039 i \text {. }
$$

The actual error is of the order of $2 \times 10^{-6}$, whereas our estimate is about $3 \times 10^{-4}$. Using five terms of the asymptotic series, we obtain the value

$$
-0.084855-0.048277 i \text {. }
$$

There is thus an actual error of about $1 \times 10^{-4}$, compared with an estimated error of about $8 \times 10^{-3}$.

\section{Remarks}

1. A third method of attack is conceivable: The integration in the complex plane of the differential equation for $E_{1}(z)$, taking $z^{-1}$ as the independent variable, extending the ideas of Fox and Miller [4].

2. It is conceivable that the Laguerre method would be effective in cases when the integrand is not precisely of the form $e^{-t} f(t)$. A case of importance might be that of the generalized exponential integral $\int^{\infty}\left(e^{-w} / w\right) d u$, where $w=\left(a^{2}+u^{2}\right)^{\frac{1}{2}}$. In such a case it would be convenient to follow the device of A. Reiz [5] and use the quadrature in the form

$$
\int_{0}^{\infty} F(t) d t \doteqdot \sum \mu_{i}^{(n)} F\left(x_{i}^{(n)}\right)
$$


where $\mu_{i}^{(n)}=\lambda_{i}^{(n)} \exp x_{i}^{(n)}$; the $\mu_{i}^{(n)}$ have been tabulated by Salzer and Zucker [2].

3. The table of Mashiko [6] gives $E_{1}(z)$, or rather auxiliary functions from which this can be obtained, for $z=r e^{i \theta}$ when $r^{-1}=0.01(0.01) 0.2$ and $\theta=0\left(2^{\circ}\right) 60^{\circ}\left(1^{\circ}\right) 90^{\circ}$.

\section{References}

[1] National Bureau of Standards, Tables of $E_{1}(z)$, (publication pending).

[2] H. E. Salzer and Ruth Zucker, Table of the zeros and weight factors of the first fifteen Laguerre polynomials, Am. Math. Soc. Bul. 55, 1004-1012 (1949).

[3] G. Szegö, Orthogonal polynomials, Amer. Math. Soc. Coll. Publications 23, 369 (1939).

[4] L. Fox and J. C. P. Miller, Table-making for large arguments. The exponential integral, MTAC 5, 163-167 (1951).

[5] A. Reiz, On the numerical solution of certain types of integral equations, Arkiv for Mat., Astron. Fysik, 29 (A); No. 29 (1943).

[6] M. Mashiko, Tables of generalized exponential-, sine-, and cosine-integrals, Numerical Computation Bureau, Report No. 7 (Tokyo,1953).

Washington, February 2, 1954. 